\title{
SEMICONDUCTOR DEVICES
}

\section{Gigahertz transducers on CMOS}

In Proc. 2019 IEEE Int. Electron Devices Meeting (in the press); https://ieee-iedm.org/program/

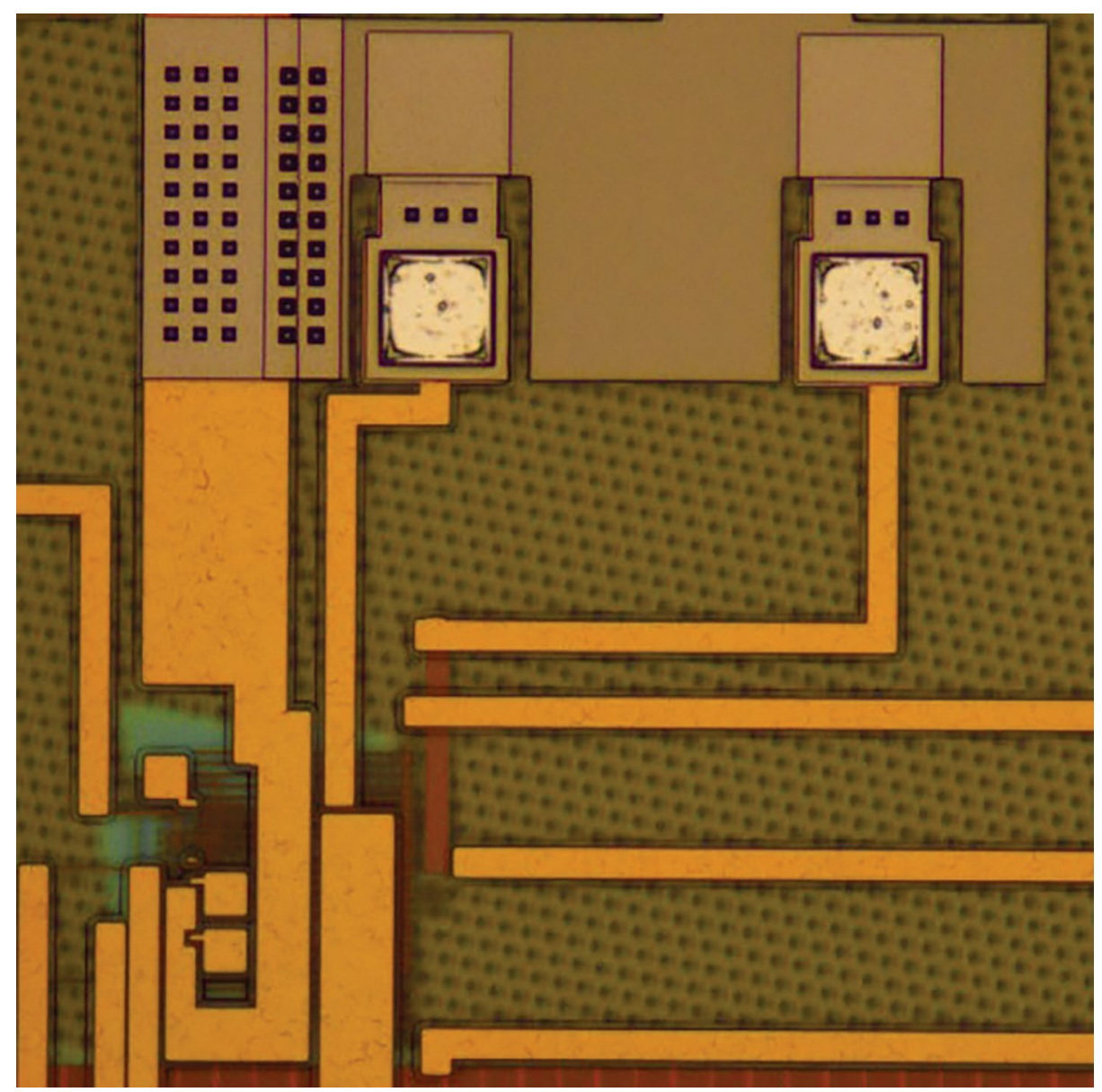

Credit: Amit Lal, Cornell University

Ultrasonic transducers that use highfrequency pulses to probe the properties of surfaces are useful for applications such as sensing, fingerprint imaging and reflectometry. The preferred piezoelectric material for ultrasonic transducers is aluminium nitride (AIN), because it is compatible with complementary metal-oxide-semiconductor (CMOS) technology and low-bias voltages. However, piezoelectric transducers have so far been incompatible with advanced CMOS nodes, require a release step during fabrication and operate at a few megahertz, limiting their imaging resolution. Amit Lal and colleagues have now developed transducers based on AlN that are monolithically integrated with CMOS electronics for gigahertz ultrasonic reflectometry.

The researchers - who are based at Cornell University and $\mathrm{A}^{\star} \mathrm{STAR}$,
Singapore - fabricated the circuits of the transducers, including the pulse transmitter and receiver, on $180 \mathrm{~nm}$ CMOS wafers, eliminating the release step. The devices can be used for sensing and actuation, either on-chip through the CMOS circuit stack or off-chip through input/output pads. Compared to a two-chip sensing system, the monolithic device consumes less power ( 3 milliwatts), can operate at lower bias voltages (less than 2 volts) and produces high-frequency pulses (up to 1.82 gigahertz). Lal and colleagues used the transducers to measure electrolyte concentrations in solution, as well as for fingerprint imaging.

\section{Christiana Varnava}

Published online: 6 December 2019 https://doi.org/10.1038/s41928-019-0348-5 